\title{
An ID-Based RFID Privacy Protocol with Weil-Pairing
}

\author{
Xie Yumin*
}

Nanjing Institute of Technology, Jiangning District, Nanjing, China

\begin{abstract}
Identification is a very important usage case of RFID. This kind of usage can be subverted by a specific type of attack, such as counterfeiting, sniffing, tracking and so on. From a certain point of view, to protect an RFID tag's privacy or security is to keep its ID hidden from an illegal reader. In this paper, we proposed an ID-based privacy protect protocol for an RFID-based smart card. This protocol is a bilinear pairings-based one, which is a lightweight public-key cryptosystem on ECC. Security attributes of the protocol are presented.
\end{abstract}

Keywords: ID-Based, RFID, Privacy, Weil-Pairing, ECC.

\section{INTRODUCTION}

Radio Frequency Identification (RFID) is an emerging technology that has been already applied to existing applications such as supply-chain management and inventory control [1]. An RFID tag is composed of a tiny microchip that is attached to an antenna. While these tags are stuck onto objects, they can be identified and tracked by readers over an electromagnetic field. RFID tags facilitate the process and the management of products during their life cycle [2].

The simplest form of an RFID system is the automatic identification of items. In general, an RFID tag will reply any well-formed reader request with their full ID. This ID can be resoled to a special issue, supply information, a product manufacturer and even a serial number [3]. If the request reader is illegal, those tags disclose the possession of certain sensitive items to them. For example, when the European Central Bank considered the use of RFID tags in Banknotes, criminal scenarios quickly surfaced in which clever robbers would screen their victims first in order to assess the amount of cash carried [4-6].

The other use case of RFID include monitoring, authentication, alerting. Due to the recent development of RFID tag technology, RFID tags can manufactured inexpensively and in non-conspicuous miniature sizes, so that they can be integrated into products during the manufacturing process of the products, rather than being attached to the packaging of the products. And at the same time RFID-tag readers are increasingly easy and inexpensive to buy. So once a specific tag has been associated with a particular item, the mere presence of this tag in a particular reader field already implies a location disclosure. Let one reader can scan a tag and let others cannot find (only presence of the tag) it is a hard difficulty.

In this paper, we proposed an ID-based privacy protect protocol for an RFID-based smart card.
The rest of the paper is organized as follows. Section 2 introduces the security problem in RFID applications. Section 3 gives basic knowledge of Weil Pairing. In section 4 we proposed our new scheme. The security of our new scheme is discussed in section 5. Section 6 concludes the paper and proposes an open problem.

\section{PROBLEM}

Typical RFID systems are composed of three elements, the RFID tag, the RFID reader and the back-end database associates records with tag data collected by readers. Unlike the cable-based communications, when the wireless tags communicate with a legal reader, any unexpected readers nearby can listen their talks in almost insensible way. This may create new threats to the security and privacy of individuals or organizations which hire the RFID application systems [10]. So some security or privacy mechanisms must be taken into consideration.

Different applications need different methods to protect their information, and further more, different RFID based systems must taken corresponding mechanisms. For example, [7] the low cost demanded for RFID tags causes them to be very resource limited. Generally speaking, these kind tags can only store hundreds of bits, and their logic gate number is often between 5000 and 10000 . Within this gate counting, only between 250 and 3000 gates can be used to security functions [7]. In this paper, we take our attention on another kind of RFID based smart card. Even we think that the RFID tag maybe attached on cell phones. If so, the limited storage and computing power on tags will be greatly improved.

Now, we give a chart to explain the idea of our algorithm. As shown in Fig. (1). Suppose a target (We call it $C$ ) equips with an RFID based smart card. It will $\log$ in a system at $A$ and $\log$ out the system at $B . I D_{C}$ is the identity of $C$. We want to use this identity to $\log$ in, but do not let or A B know the true value of it. 


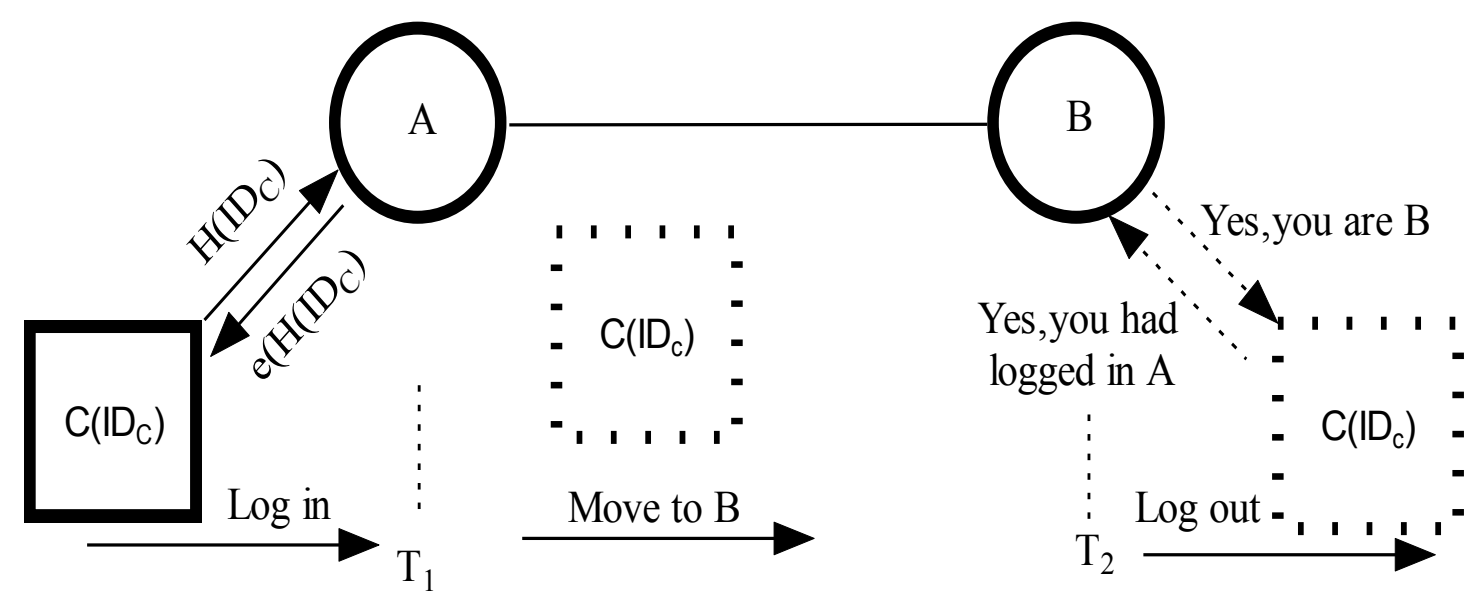

Fig. (1). The main idea of our algorithm.

After $C \log$ out the system from $B$, it can clear all information which get from $A$, and $A$ or $B$ know nothing about the true value of $I D_{C}$ during this process.

\section{BASIC KNOWLEDGE OF WEIL PAIRING}

Let $G_{1}$ be a cyclic additive group generated by $P$, whose order is a prime $q$, and let e: $G_{1} \times G_{1} \rightarrow G_{2}$ be a paring which satisfies the following conditions [11]:

A: $\quad$ Bilinear $\quad e\left(P_{1}+P_{2}, Q\right)=e\left(P_{1}, Q\right) e\left(P_{2}, Q\right) \quad$ and $e\left(P, Q_{1}+Q_{2}\right)=e\left(P, Q_{1}\right) e\left(P, Q_{2}\right)$;

B: Non-degenerate, there exists $P \in G_{1}$ and $Q \in G_{1}$ such that $e(P, Q) \neq 1$

C: Computability, there is an efficient algorithm to compute function $e$.

The m-torsion subgroup of $E$ ( $m \in Z, E$ is an elliptic curve), denoted by $E[m]$, is the set of points of order $m$ in $E: E[m]:=\{P \in E \mid m P=O\}$

$P \in E[m]$ is called an m-torsion point. Usually we choose $m$ as a prime divisor of $\# E\left(F_{P}\right)$.

Lemma 1: Suppose $E[m]$ is an m-torsion subgroup on an elliptic curve $E$, and $m$ is a prime. For any $P \in E[m]$ and $P \neq O$, there exists $2 P \neq O$ and $P / 2 \neq O$.

Proof: if there exists an element $P \in E[m]$ and $P \neq O$, which satisfies $2 P=O$, then we can find the generator of $E[m]$ is not $P$. Suppose the generator is $Q$, then there is a number $z \in Z$, and we can get $P=z Q$ and then $2 P=2 z Q=O$, finally we get a contradiction $2 z=m$.

Now we define a function $f(P)=2 P(P \neq O)$. For any $P_{1}, P_{2} \in E[m] \backslash\{O\}$ and $P_{1} \neq P_{2}$, there exists:
$f\left(P_{1}\right) \neq f\left(P_{2}\right)$

$f\left(P_{1}\right) \neq O$

$f\left(P_{2}\right) \neq O$

So $f$ is a one to one function on $E[m] \backslash\{O\}$, and according to $f^{-1}$ we can get $P / 2 \neq O$.

Lemma 2: Suppose $E[m]$ is an m-torsion subgroup on an elliptic curve $E$, and $m$ is a prime. For any $P, Q \in E[m] \backslash\{O\}$, there exists:

$e(2 P, Q / 2)=e(P, Q)$

Obviously,

$e(2 P, Q / 2)$

$=e(P, Q / 2) e(P, Q / 2)=e(P, Q / 2+Q / 2)$

$=e(P, Q)$

\section{ID-BASED PRIVACY PROTECT PROTOCOL}

General ID-based cryptography includes four algorithms: Setup, Extract, Encrypt, Decrypt. Our protocol is slightly different from general one. It includes four algorithms named Setup, Extract, Log in and Log out. Our protocol works as follows:

\section{Setup Begin}

Let $P$ be a generator of $G_{1}$ : Remember that $G_{1}$ is an additive group of prime order $q$ and the bilinear pairing is given by $e: G_{1} \times G_{1} \rightarrow G_{2}$. Define two cryptographic hash functions:

$H:\{0,1\}^{*} \rightarrow Z_{q}$

$H_{1}:\{0,1\}^{*} \rightarrow G$. 
Key Generation Center(KGC) [6] chooses a random number $s \in Z_{q}^{*}$ and set $P_{P u b}=s P$. Then it publishes system parameters:

$\left\{G, q, P, P_{P u b}, H, H_{1}\right\}$.

KGC keeps $S$ as the master-key, which is known only by itself.

\section{Setup End}

\section{Extract Begin}

Users, such as $A, B, C$ send following IDs information to KGC.

$Q_{A}=H_{1}\left(I D_{A}\right)$,

$Q_{B}=H_{1}\left(I D_{B}\right)$,

$Q_{C}=H_{1}\left(I D_{C}\right)$

$\mathrm{KGC}$ send the following messages to $A, B, C$ respectively.

$S_{A}=s Q_{A}$,

$S_{B}=s Q_{B}$,

$S_{C}=s Q_{C}$

If two readers, such as $A, B$, would to be construct a system, one acts as a log in reader, the other acts as a $\log$ out reader, they should do the following works:

$A: P_{A}=a P$,

$P_{A}^{\prime}=a^{\prime} P$

$T_{H a}=H\left(P_{A}, P_{A}^{\prime}\right)$

$T_{A e}=e\left(P_{A}, P_{A}^{\prime}\right)$

$B: P_{B}=b P$,

$P_{B}^{\prime}=b^{\prime} P$,

$T_{H b}=H\left(P_{B}, P_{B}^{\prime}\right)$,

$T_{B e}=e\left(P_{B}, P_{B}^{\prime}\right)$

$B \rightarrow A: T_{H b}, T_{B e}$

$A \rightarrow B: T_{H a}, T_{A e}$

\section{Extract End}

\section{$\mathrm{C} \log$ in A Begin}

$C \rightarrow A: Q_{C}\left(A\right.$ stores $Q_{C}$ into its DB $)$

$$
\begin{aligned}
& A: P_{C}=c P_{A} \\
& P_{C}^{\prime}=c^{\prime} P_{A}^{\prime} \\
& T_{P c}=c P_{C}^{\prime} \\
& P_{A}=2 P_{A} \\
& P_{A}^{\prime}=P_{A}^{\prime} / 2 \\
& T_{L a}=e\left(H\left(P_{A}, P_{A}^{\prime}\right) Q_{A}, P_{P u b}\right) T_{A e} e\left(P_{C}, P_{C}^{\prime}\right) \\
& T_{A}=H\left(P_{A}, P_{A}^{\prime}\right) S_{A} \oplus a P_{A}^{\prime} \\
& T_{X a}=\left(e \left(H\left(P_{A}, P_{A}^{\prime}\right) Q_{A}+\right.\right. \\
& \left.\left.T_{H b} Q_{B}, P_{P u b}\right) T_{A e} T_{B e}\right) \oplus T_{B e} \\
& A \rightarrow C: P_{C}, P_{C}^{\prime}, T_{P c}, T_{L a}, T_{A}, T_{X a} \\
& C: P_{C}=k P_{C} \\
& P_{C}^{\prime}=P_{C}^{\prime} / k \\
& T_{L c}=T_{L a} e\left(H\left(P_{C}, P_{C}^{\prime}\right) Q_{C}, P_{P u b}\right) \\
& T_{C}=H\left(P_{C}, P_{C}^{\prime}\right) S_{C} \oplus T_{P c}
\end{aligned}
$$

\section{$C \log$ in A End}

\section{C log out B Begin}

$$
\begin{aligned}
& C \rightarrow B: T_{A} \\
& \quad B \rightarrow C: \text { if }\left(\left(T_{A} \text { is not included in } \mathrm{DB} \text { of } \mathrm{B}\right)\right. \text { sends } \\
& e\left(T_{A}+T_{B}, P\right) \oplus T_{B e} \text { to } C \\
& \quad C: \text { if }\left(e\left(T_{A}+T_{B}, P\right) \oplus T_{B e}=T_{X a}\right) \text { sends } \\
& T_{C}, T_{L c} \text { to } B \\
& \left.\quad B: \text { if }\left(e\left(T_{A}+T_{C}, P\right)==T_{L c}\right)\right) \text { accepts } C \text { and adds } T_{A} \text { to }
\end{aligned}
$$
the DB of $B$.

\section{C log out B End}

\section{ANALYSIS OF THE PROTOCOL}

In fact, in our protocol we take advantage of the main idea of [8]. In this paper, the authors present a new ID-based one round authenticated tripartite key agreement protocol with pairings. Now we list its main idea as follows:

Let $\mathrm{A}, \mathrm{B}$ and $\mathrm{C}$ be the three entities who are going to agree to some session keys. After they finish the Extract step as the general ID-based one does, they do the following woks: 


$$
\begin{aligned}
& A \rightarrow B, C: P_{A}=a P, P_{A}^{\prime}=a^{\prime} P \\
& T_{A}=H\left(P_{A}, P_{A}^{\prime}\right) S_{A}+a P_{A}^{\prime} \\
& B \rightarrow A, C: P_{B}=b P, P_{B}^{\prime}=b^{\prime} P \\
& T_{B}=H\left(P_{B}, P_{B}^{\prime}\right) S_{B}+b P_{B}^{\prime} \\
& C \rightarrow A, B: P_{C}=c P, P_{C}^{\prime}=c^{\prime} P \\
& T_{C}=H\left(P_{C}, P_{C}^{\prime}\right) S_{C}+T_{P_{C}}
\end{aligned}
$$

$A$ verifies:

$$
\begin{aligned}
& e\left(T_{B}+T_{C}, P\right)=e\left(H\left(P_{B}, P_{B}^{\prime}\right) Q_{B}+\right. \\
& \left.H\left(P_{C}, P_{C}^{\prime}\right) Q_{C}, P_{P u b}\right) e\left(P_{B}, P_{B}^{\prime}\right) e\left(P_{C}, P_{C}^{\prime}\right) \\
& \quad B \text { verifies: }
\end{aligned}
$$

$$
\begin{aligned}
& e\left(T_{A}+T_{C}, P\right)=e\left(H\left(P_{A}, P_{A}^{\prime}\right) Q_{A}+\right. \\
& \left.H\left(P_{C}, P_{C}^{\prime}\right) Q_{C}, P_{P u b}\right) e\left(P_{A}, P_{A}^{\prime}\right) e\left(P_{C}, P_{C}^{\prime}\right)
\end{aligned}
$$

$C$ verifies:

$$
e\left(T_{A}+T_{B}, P\right)=e\left(H\left(P_{A}, P_{A}^{\prime}\right) Q_{A}+\right.
$$$$
\left.H\left(P_{B}, P_{B}^{\prime}\right) Q_{B}, P_{P u b}\right) e\left(P_{A}, P_{A}^{\prime}\right) e\left(P_{B}, P_{B}^{\prime}\right)
$$

If the above equations hold, $A, B, C$ can compute eight secret session keys respectively. Such as $A$ :

$$
\begin{aligned}
& K_{A}^{1}=e\left(P_{B}, P_{C}\right)^{a}\left(=K_{B}^{1}=K_{C}^{1}\right), \\
& K_{A}^{2}=e\left(P_{B}, P_{C}^{\prime}\right)^{a}\left(=K_{B}^{2}=K_{C}^{2}\right),
\end{aligned}
$$

$$
K_{A}^{8}=e\left(P_{B}^{\prime}, P_{C}^{\prime}\right)^{a^{\prime}}\left(=K_{B}^{8}=K_{C}^{8}\right)
$$

Now we give a definition first, then use this definition to analyze the security properties of out giving protocol.

Definition: The Bilinear Diffie-Hellman (BDH) Problem for a bilinear paring e: $e: G_{1} \times G_{1} \rightarrow G_{2}$ is defined as follows: given $P, a P, a^{\prime} P \in G$, compute $e\left(a P, a^{\prime} P\right)$, where $a, a^{\prime} \in Z_{q}^{*}$. An algorithm is said to solve this problem with an advantage of $\varepsilon$ if

$$
\operatorname{Pr}\left[\Re\left(P, a P, a^{\prime} P\right)=e\left(a P, a^{\prime} P\right)\right] \geq \varepsilon
$$

We know that only from $P, a P$ to get $a$ is a hard problem. We assume that BDH problem is hard [8]. And in this paper, we call these two hard problems as BDH problem.

In above protocol, after $C \log$ in the system, only $T_{A}, e\left(T_{A}+T_{B}, P\right) \oplus T_{B e}, T_{C}, T_{L c}$ are transferred under insecurity air channels.
5.1. $T_{A}$

At the extract stage, $A$ chooses random ephemeral keys $a, a^{\prime}$ from $Z_{q}^{*}$, and set $P_{A}=a P, P_{A}^{\prime}=a^{\prime} P$. When an entity $C \log$ in to the system, $A$ set $T_{A}$ as follows:

$$
\begin{aligned}
& P_{A}=2 P_{A}, P_{A}^{\prime}=P_{A}^{\prime} / 2 \\
& T_{A}=H\left(P_{A}, P_{A}^{\prime}\right) S_{A} \oplus a a^{\prime} P
\end{aligned}
$$

So after a series of entities $C_{1}, C_{2}, \cdots, C_{n}$ logged out the system, an illegal reader may get $T_{A i}$ as:

$$
T_{A i}=H\left(2 i P_{A}, P_{A}^{\prime} / 2 i\right) S_{A} \oplus a a^{\prime} P(\mathrm{i}=1,2, \ldots, \mathrm{n})
$$

Now change

$T_{A i}=H_{i} P_{G} \oplus a a^{\prime} P$ where:

$H_{i}=H\left(2 i P_{A}, P_{A} / 2 i\right)$,

$P_{G}=Q\left(I D_{A}\right) \in G$

$H_{i}, s, P_{G}, a, a^{\prime}$ are all keep as private values by $A$ according to our protocol. So obviously, if only know finite numbers $T_{A i}$, computing $a, a^{\prime}$ is a BHD problem.

5.2. $e\left(T_{A}+T_{B}, P\right) \oplus T_{B e}$

$e\left(T_{A}+T_{B}, P\right) \oplus T_{B e}=$

$e\left(T_{A}, P\right) e\left(T_{B}, P\right) \oplus T_{B e}$

$T_{B e}$ is keep as private values by $A$ and $B, T_{B}$ is keep as private values by $B$ according to our protocol. So according to the analysis of 4.1, if only know finite numbers $T_{A i}$, computing $T_{B e}$ or $T_{B}$ is a BHD problem.

\section{3. $T_{C}, T_{L c}$}

$T_{C}, T_{L c}$ are used only once after $C$ find that $B$ is the exact one it will $\log$ out. After $C$ send the two values to entity $B$, it will be accepted by $B$ if those values are legal. Then the values are no usage any more.

\subsection{A Precondition}

Following conditions should be satisfied when $A$ selects $a, a^{\prime}, c, c^{\prime}$ and $B$ selects $b, b^{\prime}$ from $Z_{q}^{*}$ :

$e\left(a P, a^{\prime} P\right) \neq 1$ 
$e\left(b P, b^{\prime} P\right) \neq 1$ and

$e\left(c P, c^{\prime} P\right) \neq 1$.

$H_{1}:\{0,1\}^{*} \rightarrow G$ can be easily converted into a normal version by using a function :

$f(m) \rightarrow h(m) P[6]$.

\section{CONCLUSION AND OPEN PROBLEM}

ID-based public key cryptosystem can be an alternative for certificate-based public key infrastructures. For key management problem, the former is more convenient then the later. In this paper, we proposed an ID-based privacy protect protocol. We suppose an RFID smart card with an identity ID would to $\log$ in and $\log$ out a system. We want to protect the card privacy while using ID as its identity to interact with the system. With our protocol, identity's ID information like the value of ID can be protected to some extent. But there still exists open problems. For example, although an adversary doesn't know the exact IDs value with an RFID card, but it can trace the value $T_{A}$ which the card attached with. So there exists I don't know who are you, but I can track you problem. In addition, like general ID-based system, there are still exist Key Escrow Problem in our protocol [9].

\section{CONFLICT OF INTEREST}

The author confirms that this article content has no conflict of interest.

\section{ACKNOWLEDGEMENTS}

Declared none.

\section{REFERENCES}

[1] B. Alomair, L. Lazos, and R. Poovendran, "Passive attacks on a class of authentication protocols for RFID", In: Information Security and Cryptology-ICISC 2007. Springer: Berlin Heidelberg, pp. 102-115, 2007.

[2] M. Hutter, Rfid Authentication Protocols Based on Elliptic Curves, Proceeding of 2009 International Conference on Security and Cryptography, pp.101-110, 2009.

[3] M. Langheinrich, "A survey of RFID privacy approaches", Personal and Ubiquitous Computing, vol. 13, no. 6, pp. 413-421, 2009.

[4] M. Langheinrich, "A survey of RFID privac approaches", Personal and Ubiquitous Computing, vol. 13, no. 6, pp. 413-421, 2009.

[5] S. Vaudenay, "On privacy models for RFID". In: Advances in Cryptology-Asiacrypt, Springer: Berlin Heidelberg, pp. 68-87, 2007.

[6] V. N. Lakshmi, I. Rameshbabu, D. L. Bhaskari, “A Security Mechanism for library management system using low cost RFID tags", Journal of Systemics, Cybernetics and Informatics, vol. 5, no. 1, pp. 92-96, 2007.

[7] P. P. Lopez, J. C. Hernandez-Castro, and J. M. Tapiador, A. Ribagorda, "RFID systems: A survey on security threats and proposed solutions", Personal Wireless Communications, pp. 159-170, 2006.

[8] F. Zhang, S. Liu, K. Kim, "ID-based one round authenticated tripartite key agreement protocol with pairings", Cryptology ePrint Archive, Report 2002/122, 2002.

[9] J. Baek, J. Newmarch, R. S. Naini, and W. Susilo, "A survey of identity-based cryptography", Proc. of Australian Unix Users Group Annual Conference, pp. 95-102, 2004.

[10] M. Kis, and E. Kalayci, "RFID Infrastructures and AI Approachs For Security", In: RFID Eurasia, $1^{\text {st }}$ Annual, pp. 1-6. 2007.

[11] X. Chen, f. Zhang, D. M. Konidala, and K. Kim, "New ID-based threshold signature scheme from bilinear pairings", In: Progress in Cryptology-INDOCRYPT, pp. 371-383, 2005.

Received: November 10, 2014

Revised: January 07, 2015

Accepted: January 19, 2015

(C) Xie Yumin; Licensee Bentham Open.

This is an open access article licensed under the terms of the Creative Commons Attribution Non-Commercial License (http://creativecommons.org/licenses/by-nc/3.0/) which permits unrestricted, non-commercial use, distribution and reproduction in any medium, provided the work is properly cited. 